\title{
One Hop-OLSR - An Efficient Technique over OLSR Routing to Maximize Energy
}

\author{
Pragya Sharma \\ M.Tech Dept. of CSE \\ LNCT \\ Bhopal, India
}

\author{
Vineet Richhariya, PhD \\ Head of Department CSE \\ LNCT \\ Bhopal, India
}

\author{
J.P. Maurya \\ Assistant Professor CSE \\ LNCT \\ Bhopal, India
}

\begin{abstract}
MANET is a wireless network, in which mobile nodes are connected, these nodes are movable in nature, thus topology of the network changes frequently. To communicate in a frequently changed scenario is a difficult task to do, an efficient routing technique is required to provide better solution for such problems. There are various routing techniques presented by the researchers. There are techniques AODV, DSDV, DSR etc. presented, which used to provide routing solution in MANET. But these technique suffers some defects like low PDR, consumes more time to deliver packet etc. OLSR (Optimized Link State Routing) uses predefined links to transmit packets that provide enhanced functionality deal with various routing issues which occurs in MANET. In that optimized links between source and destination are provided to transmit packets. But in that multiple paths to the destination is provided. To provide optimized path to the user a selection of the optimized path is required. That degrades performance of the whole technique. User need to wait for a long duration to select optimized path. A new one-hop clustering based technique is presented. In that a clustering technique is used to provide a single link for the one node to the other node. In that way an easy mechanism to search optimized route is provided by the proposed technique. A performance analysis for the proposed technique is presented in result and analysis Section which shows that proposed technique performs better as compare to the existing technique.
\end{abstract}

\section{Keywords}

MANET, OLSR Modification, PDR, DSDV.

\section{INTRODUCTION}

Mobile ad-hoc network is a wireless network in which various mobile nodes are connected. In that, these nodes are connected in a self-configure and a self-optimized, works as a mobile routers and mobile hosts. Thus because of the movable nature of the mobile nodes, design and topology of the networks changes frequently. To route packet from source to destination is a difficult task to do. There various challenges like low packet delivery ratio, loss of packets, and congestion in the transmission channels, low battery life, high end to end delay etc. which requires an efficient routing mechanism to handle such issues. There are various techniques like AODV (Ad-Hoc on-demand distance vector), DSR, AM route etc. are presented by the various researchers to provide efficient solutions to resolve these issues. But an advancement is still required to provide better routing solution to route packet.

OLSR (Optimized Link State Routing) is a routing technique which is generally used to resolve such issues. In that technique a predefined the information about the links to the other nodes is presented which provides an enhanced functionality to route the packet. An optimized route selection mechanism to select an optimized route to deliver packet in MANET. But in case of a multi hop route scenario multiple links from one node to the other are there. To select an optimized path, is a difficult task to do. And it also generate a maximize delay, so user need to wait for a long time to select an optimized path to transmit packet.

A one hope clustering based routing mechanism for OLSR is presented in this paper. In that clustering of the node is performed in a way, no node have more than one link to the other node, in that way it reduces the time to select optimized route to transmit packet. Also improve the performance of the technique by reducing the time consume to transmit packet.

Rest of the paper organizes as follows:

II Related Work, related work for which used clustering to improve the routing functionality in MANET is presented. III Problem Definition problems with the existing technique are discussed in this section. IV Proposed Technique, a description for the proposed technique is presented in this section. V Result Analysis, comparison analysis for the proposed technique is presented in this section. VI Conclusion.

\section{RELATED WORK}

In our recent paper the technique OLSR is introduced where a routing matrix introduced along with the existing standard OLSR with which the following concepts were added for reducing energy consumption at each node to get maximum output from a network in wireless sensor network communication. Packet transmission and Overhearing from the neighbor nodes are two important factors which consider for the energy efficient routing according to their work. They have utilized three routing approach namely 1 . MAC queue utilization 2. Residual energy, 3. Node degree. They have changed the proactive manner of standard OLSR and TC packet format in order to make integrated routing scheme. They have used Greedy Heuristic Algorithm for the routing table computation for efficient routing scheme in Enhanced OLSR work done by them. And in future they kept a work in large network to make a network system fast due to their changes done in TC packets. Hence robustness of system can be done by proceeding the work.

In the field of MANET for increasing the network lifetime by reducing energy usability in the network routing scenario, they have investigates and performed the research to find reason behind energy depletion and how can mitigate from it. They have proposed multi metric scheme which was integrated with the existing algorithm OLSR using their routing approach. Authors performed simulation using NS3 environment where they have shown the efficiency in the terms of packet delivery ratio (PDR) which outperform 10$20 \%$ more efficient than the existing standard OLSR technique. 


\section{PROBLEM DEFINITION}

In the recent work it has noticed that the work done with the less packet numbers, low traffic and weight given onto the nodes and thus the observation can't be monitor according to those steps. In the further work we have observed some problem definitions-

1. Energy efficient routing is not performed using any energy peak value.

2. Optimized link is not observed, thus it generate high packet loss which leads to low residual energy.

3. Low Packet delivery ratio in existing algorithm due to node drop as no optimized algorithm is performed in between node communication.

\section{PROPOSED WORK}

In existing technique there are various algorithms are presented which uses OLSR protocol to transmit packets as mention in problem definition, there are various variants are presents in nature to provide better performance in routing in MANET.

A new one hop clustering based OLSR routing technique is presented which provides an enhanced functionality to route packets in MANET. In that all nodes are clustered in a manner in which each node having a single link to the other nod and by the use of cluster head selection algorithm. The cluster which contains highest energy is marked as cluster head. Last information of the node are compared if it matched then that information send to the next array. Then the comparison of the node's array is conducted if two or more than two values are matched then comparison for speed is performed, node which contains higher speed is considered as cluster head.

Head notification. :

i) Broadcast hello packets in network (Weight).

ii) Receive replies

iii) Put packet in a table $\left[\mathrm{W}_{\mathrm{I}}\right]$.

iv) if $\left(\mathrm{W}<\mathrm{a}\left[\mathrm{W}_{\mathrm{i}}\right]\right)$

v) Wait for Cluster Head Declaration

vi) Else

vii) Broadcast its address as Cluster Head

Input Module

Any data packet comes to the node, check for its source address and cluster head address is conducted. If the source address same as the cluster head address then that packet consider to send to the destination, else discarded.

Following procedure is used to send packets
i) Extract input data id (CLUSTER HEAD ADDRESS)
ii) Match input data id with cluster id.
iii) If (cluster id = input id)
iv) Discard related entry from regarding tables.
v) Else
vi) Put entry in regarding table.

Flow Diagram for the proposed Technique.

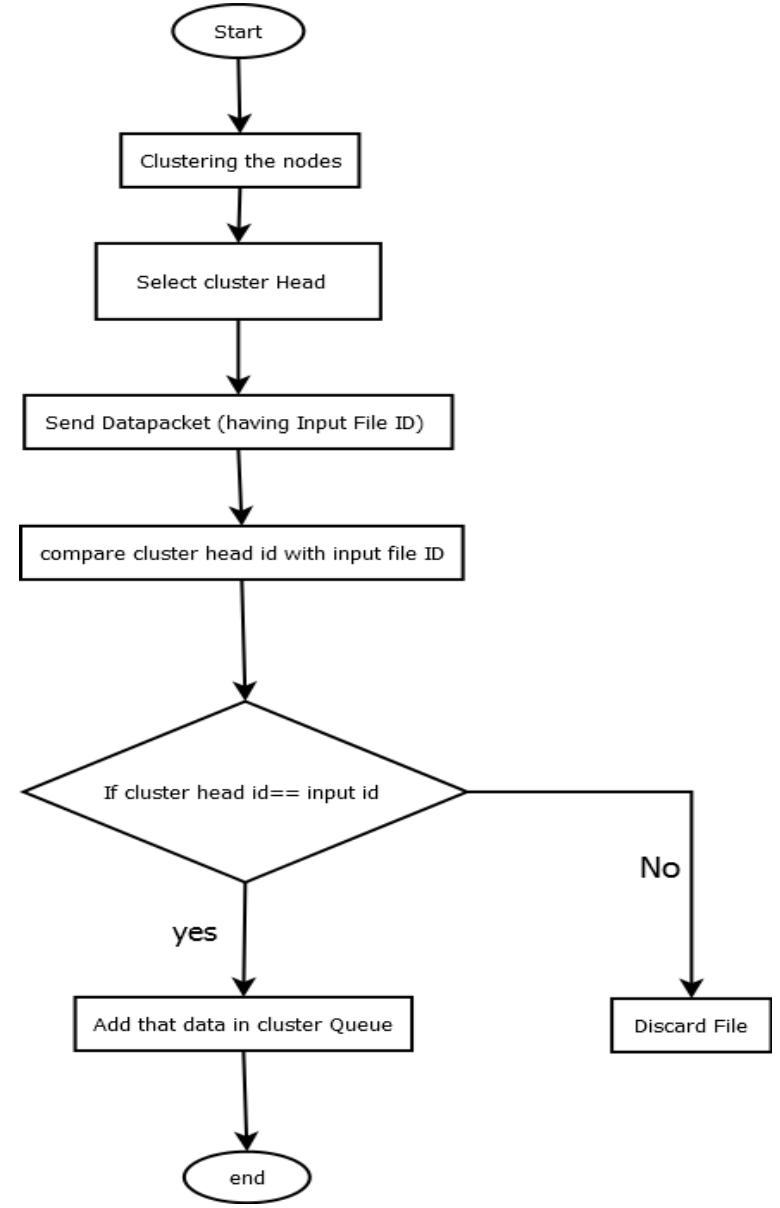

Figure 5.1: Flow Diagram for Proposed Technique.

\section{RESULT ANALYSIS}

To implement the proposed technique, a NS2 simulator is used. It is a tool which used to provide development environment for the various research in communication network.

To evaluate the performance of the proposed technique, both the techniques are run is the same environment and then a comparison analysis over the parameters called normalized routing load, average delay, Throughput, packet delivery ratio is conducted. Which shows that proposed one hop clustering based technique provides better results as compare to the existing technique.

Simulation scenario for the proposed technique in shown below.

Table: Simulation Scenarios

\begin{tabular}{|l|l|}
\hline No. of Nodes & 30 \\
\hline No. of Source & 10 \\
\hline Area & $1000 \mathrm{X} 1000$ \\
\hline $\begin{array}{l}\text { Mobility } \\
\text { model }\end{array}$ & Random waypoint \\
\hline Bandwidth & $2 \mathrm{mbps}$ \\
\hline Speed & $0,1,5,10,15,20 \mathrm{~m} / \mathrm{s}$ \\
\hline Pause time & $10 \mathrm{sec}$ \\
\hline Buffer Size & 100 \\
\hline
\end{tabular}




\begin{tabular}{|l|l|}
\hline $\begin{array}{l}\text { Transmission } \\
\text { range }\end{array}$ & $2100 \mathrm{~m}$ \\
\hline Sensing range & $2100 \mathrm{~m}$ \\
\hline Packet size & 1012 bytes \\
\hline Traffic source & CBR \\
\hline MAC protocol & IEEE 802.11 \\
\hline
\end{tabular}

Normalized Routing Load (NRL) Vs. Speed

This is all results in increase in NRL.

Table: Normalized Routing Load Vs. Speed.

\begin{tabular}{|c|l|l|}
\hline Speed(m/s) & OLSR & Modified OLSR \\
\hline $\mathbf{0}$ & 0.46 & 0.37 \\
\hline $\mathbf{1}$ & 0.37 & 0.33 \\
\hline $\mathbf{5}$ & 0.89 & 0.78 \\
\hline $\mathbf{1 0}$ & 3.99 & 3.11 \\
\hline $\mathbf{1 5}$ & 6.02 & 6.16 \\
\hline $\mathbf{2 0}$ & 4.04 & 3.99 \\
\hline
\end{tabular}

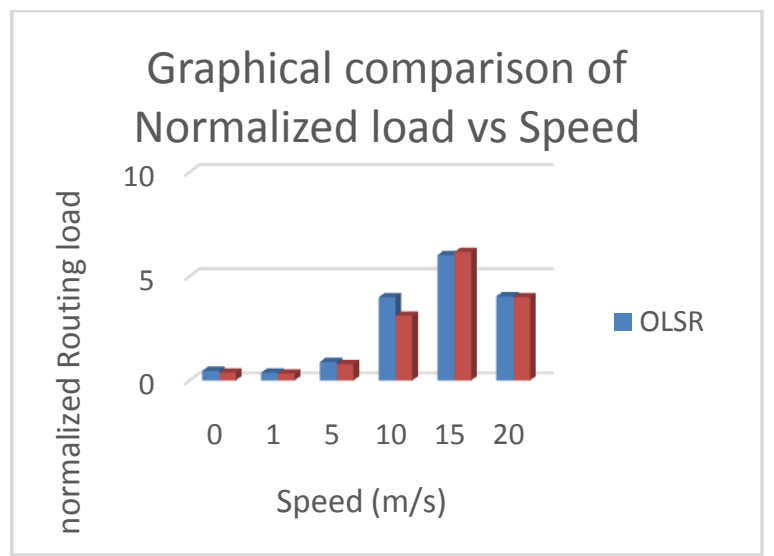

Figure 6.1: A graph for normalize route vs. mobility.Throughput vs. Speed

As PDR increases, the impact is clearly on throughput that increases too.

Table: Throughput vs. Speed

\begin{tabular}{|c|l|l|}
\hline Speed(m/s) & \multicolumn{1}{|c|}{ OLSR } & Modified OLSR \\
\hline $\mathbf{0}$ & 76.89 & 78.46 \\
\hline $\mathbf{1}$ & 77.27 & 80.00 \\
\hline $\mathbf{5}$ & 73.26 & 75.60 \\
\hline $\mathbf{1 0}$ & 70.32 & 71.63 \\
\hline $\mathbf{1 5}$ & 67.47 & 69.44 \\
\hline $\mathbf{2 0}$ & 61.28 & 62.81 \\
\hline
\end{tabular}

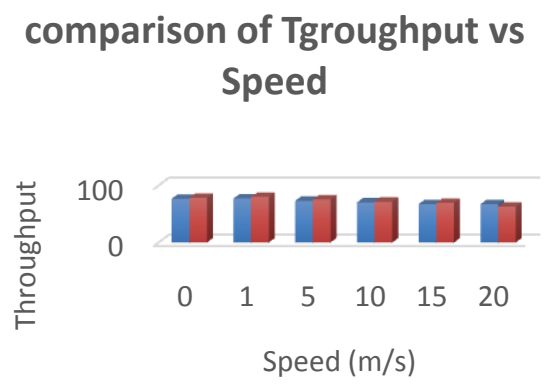

- Series 1 Series 2

Figure 5.2: Throughput Vs. Mobility speed. Packet Delivery Ratio (PDR) Vs. Speed

Because of multiple paths used for transmission, packet delivery ratio increases as chances of packet loss due to path breaks reduces. Clearly, modified OLSR performs better than the original one.

Table: Packet Delivery Ratio (PDR) Vs. Speed.

\begin{tabular}{|c|l|l|}
\hline Speed(m/s) & OLSR & $\begin{array}{c}\text { Modified } \\
\text { OLSR }\end{array}$ \\
\hline $\mathbf{0}$ & 82.77 & 85.93 \\
\hline $\mathbf{1}$ & 81.54 & 86.54 \\
\hline $\mathbf{5}$ & 75.27 & 79.60 \\
\hline $\mathbf{1 0}$ & 73.19 & 73.89 \\
\hline $\mathbf{1 5}$ & 72.49 & 74.33 \\
\hline $\mathbf{2 0}$ & 69.59 & 75.49 \\
\hline
\end{tabular}

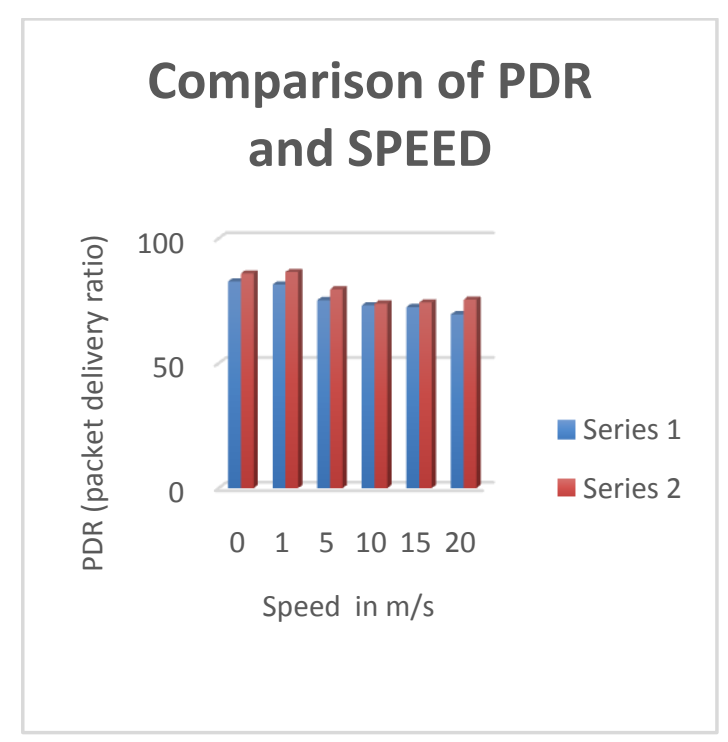

Figure 6.3: Packet delivery ratio vs. Mobility Speed.Average Delay vs. Speed

Because of multiple path features in modified OLSR, packets need not to wait for long. Also, packets are moving from 10 paths which means apart from shortest path some other paths are also followed. As a result, packet faced less queuing delay 
whereas original OLSR followed shortest route which increases queuing delay.

Table: Average Delay vs. Speed

\begin{tabular}{|c|c|c|}
\hline Speed(m/s) & OLSR & Modified OLSR \\
\hline $\mathbf{0}$ & 29.20 & 25.41 \\
\hline $\mathbf{1}$ & 20.84 & 19.42 \\
\hline $\mathbf{5}$ & 24.95 & 23.15 \\
\hline $\mathbf{1 0}$ & 45.12 & 41.17 \\
\hline $\mathbf{1 5}$ & 56.20 & 55.65 \\
\hline $\mathbf{2 0}$ & 69.96 & 69.03 \\
\hline
\end{tabular}

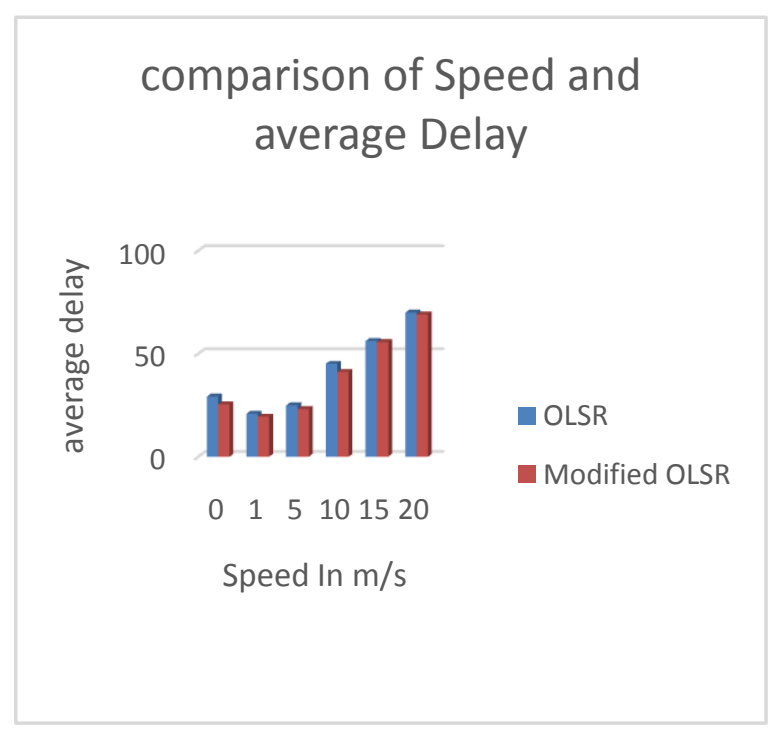

Figure 6.4: End-to End Delay vs. Mobility Speed.

As per the observation using graph monitoring here we state that the proposed algorithm generate an optimized path, also it given the high packet delivery ratio and less packet loss, thus the protocol presented is energy efficient in its execution.

\section{CONCLUSION}

Mobile ad-hoc network is a network in which multiple mobile nodes are connected over a wireless channel to communicate to one another. Ad -hoc network is an infrastructure less network which provides structure less communication mechanism to the user. In MANET design and topology of the network changes frequently which requires efficient routing technique to provide a better routing mechanism in that continuous changing scenario. OLSR (optimized link state routing technique) is generally used to provide routing mechanism to route packet from source to destination. But there are multiple links a connected to the node, thus a selection for the optimized link is required which increases the delay and degrades the per4formance of the technique. A comparison analysis for the results is shown in result and analysis section which shows that proposed technique provides better results as compare to the existing technique, in that an efficient routing mechanism is provided to route packet in the MANET.

\section{REFERENCES}

[1]. Euripides Paraskevas, Kyriakos Manousakis, Subir Dasand John S. Baras in paper "Multi-Metric Energy Efficient Routing in Mobile Ad-Hoc Networks"2014 IEEE Military Communications Conference.

[2]. P.Purniemaa, K. Manikandan, M.A.Saleem Durai; "A Framework for Securing Enhancement in Mobile-Adhoc Network", (IJCST) Internal Journal of Computer Science and Technology, Vol.2, issue 2, June 2011, PP: 118-122.

[3]. S.A.Adel and P.A Tijare, "performance Comparison of AODV, DSDV, OLSR and DSR Routing protocols in Mobile Ad Hoc Networks", International Journal of Information Technology and Knowledge Management, July-Dec 2010.

[4]. . S.Tamilarasan, "A Performance Analysis of Multihop Wireless Ad-Hoc Network Routing Protocols in MANET", International Journal of Computer science and Information Technologies (IJCSIT), Vol 2 (5), 2011, PP: 2141-2146.

[5]. Thodeti Srikanth, et al, "Simulation-based approach to performance study of routing protocol in MANETs and ad-hoc network", IJCSNS, vol.11no.9, September 2011.

[6]. F. De Rango and M. Fotino, "Energy Efficient OLSR Performance Evaluation Under Energy Aware Metrics," in Proceedings of the 12th International Conference on Symposium on Performance Evaluation of Computer \& Telecommunication Systems, ser. SPECTS'09. Piscataway, NJ, USA: IEEE Press, 2009, pp. 193-198.

[7]. S. Mahmoud and P. Mine, "An Energy Efficient Routing Based on OLSR in Wireless Ad Hoc and Sensor Networks," in Advanced Information Networking and Applications - Workshops, 2008. AINAW 2008. 22nd International Conference on, March 2008, pp. 12531259

[8]. YI GAO \& Wei Dong "COPE: Improving Energy Efficiency with Coded Preambles in Low Power Sensor Networks" 2015, IEEE Transaction and Industrial Informatics.

[9]. Nguyen Duy Tan and Nguyen Dinh Viet "Sleep scheduled and Tree -Based Clustering Routing Protocol for Energy -Efficient in Wireless Sensor Networks"2015, International Conference on Computing \& Communication Technologies.

[10].Sonali Sen Baidya and Abhishek Baidya "Energy Conservation in a wireless Sensor Network by an efficient Routing Mechanism" 2015 International conference on communication, Information \& Computing Technology. 